\section{Does Chemoradiation Benefit Patients With Gastric Cancer Managed Without Surgery?}

\author{
Kimberly L. Johung, MD, PhD, and Stacey M. Stein, MD
}

Curative-intent treatment for gastric cancer requires surgical resection with a subtotal or total gastrectomy and regional lymph node dissection, except for in carcinoma in situ or T1a tumors, select cases of which can be managed with endoscopic therapy. Phase III randomized trials published more than a decade ago established 2 standard-of-care approaches for the management of locally advanced gastric cancer. The UK MAGIC trial showed a survival benefit with perioperative chemotherapy consisting of epirubicin/ cisplatin/fluorouracil (ECF) compared with surgery alone, ${ }^{1}$ and the Intergroup 0116 trial showed a survival benefit with postoperative chemoradiation (CRT) to 45 Gy and concurrent fluorouracil and leucovorin. ${ }^{2}$

More recently, the CRITICS trial compared these treatment strategies. In patients who received preoperative chemotherapy and adequate surgery, no survival benefit was seen with postoperative CRT compared with postoperative chemotherapy. ${ }^{3}$ Treatment strategies further evolved after the FLOT4-AIO trial was presented at the 2017 ASCO Annual Meeting. Perioperative ECF was compared with perioperative FLOT (docetaxel/ oxaliplatin/fluorouracil/leucovorin), with median overall survival (OS) improving from 35 to 50 months with FLOT. ${ }^{4}$ Thus, perioperative FLOT has emerged as a standard treatment approach for gastric cancer.

Although these and other phase III trials define the optimal management of resectable gastric cancer, ${ }^{1-6}$ data are lacking to guide treatment for the large percentage of patients who do not undergo surgery. An analysis of the National Cancer Database (NCDB), which included $>45,000$ patients with stage IA-IIIC gastric adenocarcinoma diagnosed between 2004 and 2013, showed that 39\% of patients did not receive curative-intent surgery. ${ }^{7}$ Factors independently associated with lack of surgery included type of insurance (not private or Medicare), treatment at a nonacademic hospital, residing in a small urban population or area with low median income, older age, and higher Charlson-Deyo score. Thus, the population of patients with gastric cancer managed without surgery includes those who face challenges regarding access to care, elderly patients, or those with medical comorbidities. Studies evaluating the optimal nonsurgical management of gastric cancer can improve outcomes for these patients.

The NCCN Clinical Practice Guidelines in Oncology (NCCN Guidelines) for Gastric Cancer recommend CRT or palliative management for patients with gastric cancer who are not surgical candidates. ${ }^{8}$ Data in support of CRT using modern techniques are limited to small, single-arm, phase II studies, including one from China in which 36 patients received 2 cycles of docetaxel/cisplatin/fluorouracil before and after intensitymodulated radiation therapy (IMRT) to 50.4 Gy with concurrent docetaxel. ${ }^{9}$ In this study, $36 \%$ of patients experienced a clinical complete response (CR) with this approach.

In the absence of larger studies or randomized data, Li et al analyzed the NCDB to compare survival for patients with localized gastric cancer treated with chemotherapy or CRT, without surgery. In their article, elsewhere in this issue, they describe their cohort of nearly 4,800 patients with nonmetastatic gastric adenocarcinoma diagnosed between 2004 and 2015. Patients with in situ or T1a disease were excluded, as were those who received any type of gastric surgery. Using multivariate analysis and propensity scorematched analysis, an OS benefit was seen with concurrent CRT or sequential chemotherapy and radiation therapy (RT) compared with chemotherapy alone.

The survival benefit $\mathrm{Li}$ et al observed with the addition of RT was modest (median OS, 12.3 vs 11.3 months for chemotherapy). RT was delivered to a median dose of $45 \mathrm{~Gy}$, and those who received $<45$ Gy had decreased survival, suggesting that a palliative dose

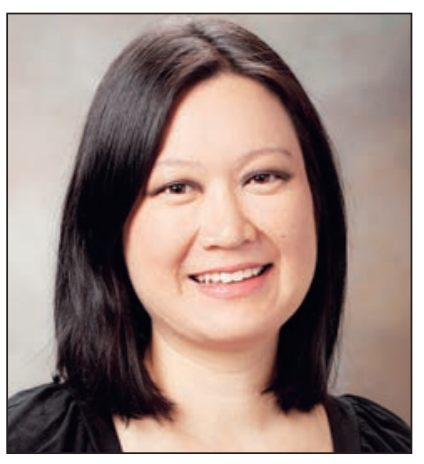

Kimberly L. Johung, MD, PhD

Kimberly L. Johung, MD, PhD, is an Assistant Professor in the Department of Therapeutic Radiology at Yale School of Medicine, and Director of the Gastrointestinal Radiotherapy Program at Smilow Cancer Hospital at Yale New Haven Hospital. She is a member of the NCCN Guidelines Panels for Esophageal and Esophagogastric Junction Cancers, Gastric Cancers, and Cancer in People Living With HIV.

doi: 10.6004/jncen.2018.7062

The ideas and viewpoints expressed in this commentary are those of the author and do not necessarily represent any policy, position, or program of NCCN. 


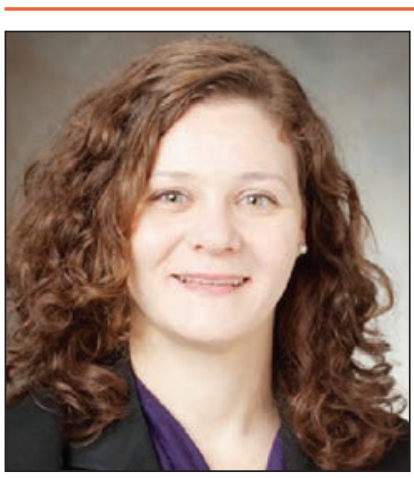

Stacey M. Stein, MD

Stacey M. Stein, MD, is an Assistant Professor in the Department of Internal Medicine, Section of Medical Oncology at Yale School of Medicine. She specializes in the treatment of gastrointestinal malignancies. She serves as a member of the NCCN Guidelines Panel for Hepatobiliary Cancers and is a member of the $\mathrm{NCl}$ hepatobiliary taskforce. of radiation was less effective at impacting outcomes. In addition, RT was delivered with concurrent chemotherapy in nearly two-thirds of patients and sequentially for the remaining third. Thus, the heterogeneity of the treatment regimen could dilute the impact of RT on patient outcomes, and it is possible that a greater benefit with RT would be seen in the setting of definitive-intent radiation doses and/or concurrent chemotherapy. Furthermore, on subset analysis, the survival benefit persisted in patients with stage I-II disease but was not significant in those with stage III disease. This result is probably because patients with stage III disease are at higher risk for metastatic progression and are less likely to benefit from locoregional therapy.

CRT can be potentially curative for patients with esophageal cancer, yet studies evaluating definitive CRT in gastric cancer are lacking. Phase II studies of preoperative RT for gastric adenocarcinoma have shown promising pathologic CR rates similar to those seen in patients with esophageal adenocarcinoma. ${ }^{10,11}$ In the RTOG 9904 study, patients received 2 cycles of induction chemotherapy followed by CRT to 45 Gy, with concurrent fluorouracil and paclitaxel. A pathologic CR was achieved in $26 \%$ of patients, with improved survival in these patients. ${ }^{11}$

The ongoing phase III TOPGEAR trial is evaluating the addition of preoperative CRT to perioperative chemotherapy in a randomized setting. ${ }^{12}$ If pathologic response and outcomes are improved with neoadjuvant CRT, results would support the consideration of CRT in the management of nonsurgical patients as well.

Retrospective large data set analyses have their inherent limitations, the most relevant being the lack of information regarding treatment intent. Nonetheless, the study by Li et al highlights the fact that a large fraction of patients with gastric cancer do not receive surgery, and that the optimal management of these patients should be better studied. Certainly, RT can provide a palliative benefit, including control of bleeding or improvement of obstructive symptoms. This analysis of the NCDB suggests that RT may improve survival outcomes for patients with unresected gastric cancer and that consideration should be given to incorporating local therapy with RT into future clinical trials for patients with unresectable gastric cancer.

\section{References}

1. Cunningham D, Allum WH, Stenning SP, et al. Perioperative chemotherapy versus surgery alone for resectable gastroesophageal cancer. N Engl J Med 2006;355:11-20.

2. Macdonald JS, Smalley SR, Benedetti J, et al. Chemoradiotherapy after surgery compared with surgery alone for adenocarcinoma of the stomach or gastroesophageal junction. N Engl J Med 2001;345:725-730.

3. Cats A, Jansen EP, van Grieken NC, et al. Chemotherapy versus chemoradiotherapy after surgery and preoperative chemotherapy for resectable gastric cancer (CRITICS): an international, open-label, randomised phase 3 trial. Lancet Oncol 2018;19:616-628.

4. Al-Batran SE, Homann N, Schmalenberg H, et al. Perioperative chemotherapy with docetaxel, oxaliplatin, and fluorouracil/leucovorin (FLOT) versus epirubicin, cisplatin, and fluorouracil or capecitabine (ECF/ECX) for resectable gastric or gastroesophageal junction (GEJ) adenocarcinoma (FLOT4-AIO): a multicenter, randomized phase 3 trial [abstract]. J Clin Oncol 2017;35(15 Suppl):Abstract 4004.

5. Park SH, Sohn TS, Lee J, et al. Phase III trial to compare adjuvant chemotherapy with capecitabine and cisplatin versus concurrent chemoradiotherapy in gastric cancer: final report of the adjuvant chemoradiotherapy in stomach tumors trial, including survival and subset analyses. J Clin Oncol 2015;33:3130-3136.

6. Noh SH, Park SR, Yang HK, et al. Adjuvant capecitabine plus oxaliplatin for gastric cancer after D2 gastrectomy (CLASSIC): 5-year follow-up of an open-label, randomised phase 3 trial. Lancet Oncol 2014;15:1389-1396.

7. Frohman HA, Martin JT, Le AT, et al. Failure to operate on resectable gastric cancer: implications for policy changes and regionalization. J Surg Res 2017;214:229-239.

8. Ajani JA, D'Amico TA, Baggstrom M, et al. NCCN Clinical Practice Guidelines in Oncology: Gastric Cancer. Version 2.2018. Accessed July 23, 2018. To view the most recent version of these guidelines, visit NCCN.org.

9. Liu Y, Zhao G, Xu Y, et al. Multicenter phase 2 study of peri-irradiation chemotherapy plus intensity modulated radiation therapy with concurrent weekly docetaxel for inoperable or medically unresectable nonmetastatic gastric cancer. Int J Radiat Oncol Biol Phys 2017;98:1096-1105.

10. Ajani JA, Mansfield PF, Janjan N, et al. Multi-institutional trial of preoperative chemoradiotherapy in patients with potentially resectable gastric carcinoma. J Clin Oncol 2004;22:2774-2780.

11. Ajani JA, Winter K, Okawara GS, et al. Phase II trial of preoperative chemoradiation in patients with localized gastric adenocarcinoma (RTOG 9904): quality of combined modality therapy and pathologic response. J Clin Oncol 2006;24:3953-3958.

12. Leong T, Smithers BM, Haustermans K, et al. TOPGEAR: a randomized, phase III trial of perioperative ECF chemotherapy with or without preoperative chemoradiation for resectable gastric cancer: interim results from an international, Intergroup trial of the AGITG, TROG, EORTC and CCTG. Ann Surg Oncol 2017;24:2252-2258. 